EPJ Web of Conferences 75, 06006 (2014)

DOI: $10.1051 /$ epjconf/20147506006

(c) Owned by the authors, published by EDP Sciences, 2014

\title{
Thin-Film Microtransformer for High Frequency Power Applications
}

\author{
Dragan Dinulovic ${ }^{1, \mathrm{a}}$, Alexander Gerfer ${ }^{1}$, Oliver Opitz ${ }^{1}$, Matthias Kaiser ${ }^{2}$, Marc C. Wurz ${ }^{2}$ and Lutz Rissing ${ }^{2}$ \\ ${ }^{1}$ Würth Elektronik eiSos GmbH Co. KG, Parkring 29, 85748 Garching, Germany \\ ${ }^{2}$ IMPT (Institute for Micro Production Technology), Leibniz Universität Hannover, An der Universität 2, 30823 Garbsen, \\ Germany
}

\begin{abstract}
This paper describes a development of a microtransformer device fabricated using thin film technology. The device is designed for higher switching frequencies beyond to $50 \mathrm{MHz}$ power applications. A especially by the microtransformer is a design, which allows wide flexibility of a device by choosing a different values of an inductance and of a windings ratio. The microtransformer device is integrated on silicon substrate consisting of a closed magnetic core and six coils. Both, primary and secondary device side consist three coils. Therefore, this design allows using of a device for different switching frequencies. As a magnetic material for transformer core a permalloy $\mathrm{NiFe} 45 / 55$ was chosen.
\end{abstract}

\section{Introduction}

A permanent increase of a switching frequency of an electronic power circuit sets new requirements for inductive components. Inductors and transformers should provide smaller inductivity and smaller size [1, 2]. For a switching frequency range between $10 \mathrm{MHz}$ and $30 \mathrm{MHz}$ a proposed inductivity of transformers should be between $100 \mathrm{nH}$ and $300 \mathrm{nH}$ and for inductors between $20 \mathrm{nH}$ and $200 \mathrm{nH}$. Decreasing of inductivity causes a decreasing of the inductor size and of the inductor profile height. However, small device sizes like 1008, 0805, 0603 (EIA size) are needed. Also the device profile becomes less as $0.5 \mathrm{~mm}$ or $0.3 \mathrm{~mm}$ [3]. Only the new fabrication technology as thin-film technology allows a fulfillment of all these requirements.

\section{Design}

A design of a developed microtransformer is shown in the Figure 1. The microtransformer consists of a closed $\mathrm{NiFe}$ magnetic core and six coils. Three coils are placed on the primary (Coil P1 - Coil P3) and three coils on the secondary transformer side (Coil S1 - Coil S3). All of six coils have a same design with equal number of windings and equal cross-section of winding. Coils on primary and secondary transformer side are connected in the series. The device shows in total 8 contacting pads ( 4 contacting pads on both transformer sides). This kind of contacting technique enables a widespread flexibility of this microtransformer device [4]. Each coil can be separately powered, therefore the transformer ratio and inductivity of the device is adjustable.
With adequate combination of powered coils it is possible to achieve different transformer ratio $(1: 1,1: 2,1: 3,2: 1$, $2: 2,2: 3,3: 1,3: 2$, and $3: 3$ ).

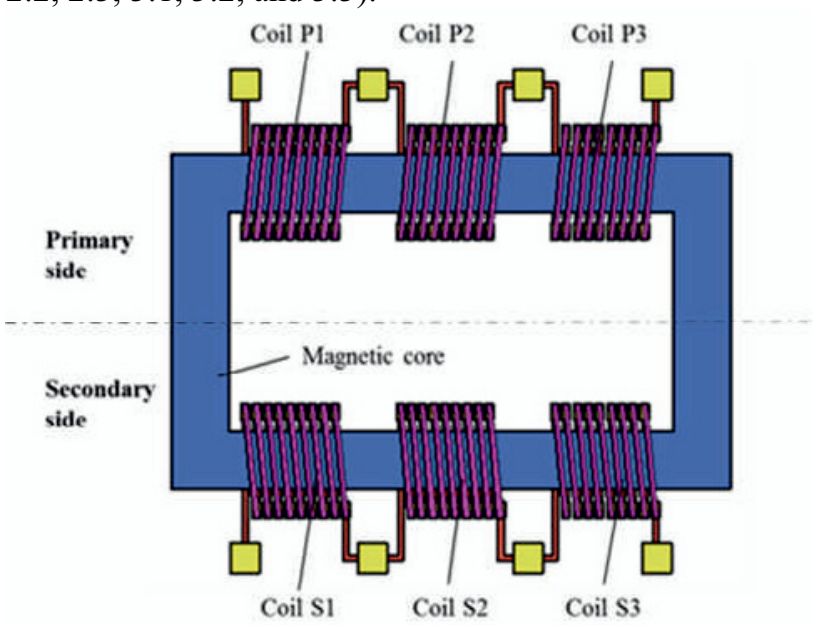

Figure 1. Schematic of microtransformer.

On this way, it is possible with only one device to cover wide range of switching frequencies i.e. by voltage conversion applications. In case of very high switching frequencies only one coil will be powered. At smaller frequencies two or three coils will be powered.

The microtransformer device can be also used as a inductor. For these applications is also possible a high flexibility, but the contacting of the pads should be modified (contacting pad of Coil P3 should be connected to pad of Coil S3). On this way, up to six coils can be connected and powered. Therefore, this design enables an adjustment of inductivity. Only with one device a wide spectrum of inductivity can be covered, and fabrication

\footnotetext{
${ }^{\mathrm{a}}$ Corresponding author: dragan.dinulovic@we-online.de
} 
costs for inductor devices with different values of inductivity can be reduced.

\section{Fabrication}

The design of a microtransformer was simulated and optimized using Finite Element Method (FEM). The software tool Ansys Maxwell ${ }^{\circledR}$ was applied. To find an optimum between design and technology issues, the technological aspects of the thin-film fabrication also have to be taken into account during the simulations. As an optimal design for magnetic core an oval core form was defined. The size of coils was restricted by complete size (length) of a microtransformer device. As a complete size of a microtransformer an EIA standard size 1008 was chosen. Therefore, whole microtransformer device features a footprint of $2.5 \mathrm{~mm} \times 2 \mathrm{~mm}$. Based on fabrication parameters (i.e aspect ratio and flank angle, defined by the photolithography processes employed) and available device size, the optimal coil design was defined. The coil consists of 9 turns. The cross-section of a coil turn is $20 \mu \mathrm{m} \times 15 \mu \mathrm{m}$.

As a magnetic material for the transformer core, the permalloy $\mathrm{NiFe} 45 / 55$ was chosen since previous investigations proved the material's suitability for magnetic MEMS applications [5], [6]. NiFe45/55 features saturation flux density $B_{s}$ of $1.6 \mathrm{~T}$ and relative high magnetic permeability $\mu_{\mathrm{r}}$. The thickness of a magnetic core should be very thin to avoid core losses. Therefore, a magnetic core should have a thickness of some $\mu \mathrm{m}$.

The micro transformer is fabricated under cleanroom conditions using high aspect ratio microstructure technology (HARMST), combining UV depth lithography and electroplating [7], [8]. A 4", silicon oxide wafer is used as substrate material. At first a seed layer stack consisting of $50 \mathrm{~nm}$ chromium and $200 \mathrm{~nm}$ gold is applied on the substrate by sputter deposition. The coil's first level consists of electroplated copper and is integrated in a coating form made of AZ9260 TM. The loops are not arranged in-plane but perpendicular to the plane shown in Figure 2.

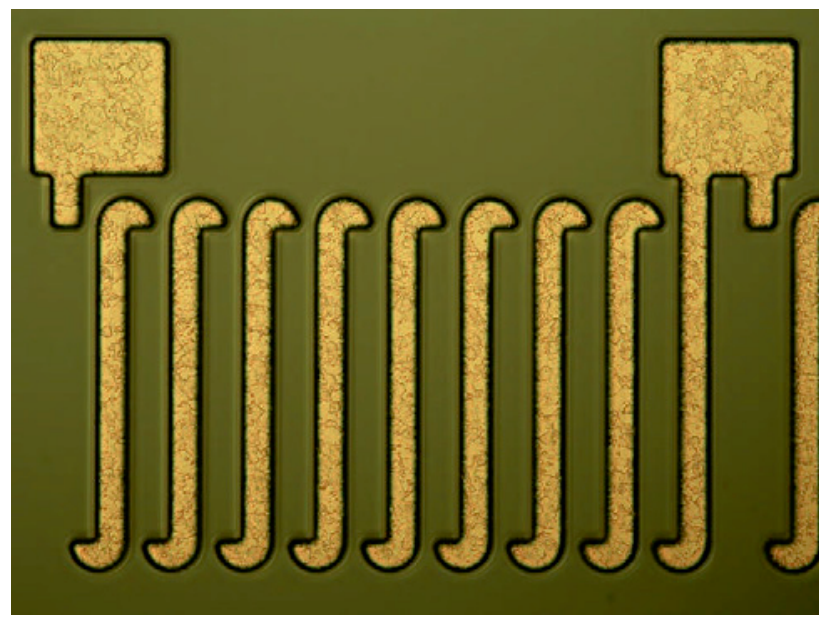

Figure 2. First layer of the copper coils.
The layer of the lower coil is $20 \mu \mathrm{m}$ thick. After the electro-plating process of the vias, the seed layer is removed by ion beam etching.

Durimid $7320 \AA$ manufactured by Fuji is deposited by spin coating serving as embedding and insulating layer. The dissolver is removed during a soft bake over two steps. The first soft bake is for 15 minutes at $70^{\circ} \mathrm{C}$ and subsequent 15 minutes at $100^{\circ} \mathrm{C}$. After the wafer has been cooled down to room temperature, the Durimid is structured in a photolithographic process. During the following hard bake at $350^{\circ} \mathrm{C}$ for 1 hour, the resin in the resist film crosslinks. Meanwhile the Durimid's height reduces from $52 \mu \mathrm{m}$ to $26 \mu \mathrm{m}$ causing distortions above the copper structures. These are removed by Chemical Mechanical Polishing (CMP), which is essential to create an even surface that is again necessary for the upcoming process steps.

The Isolation material's height and the aspect ratio do not allow developing the Vias in a chemical solution. Thus an additional process step is established: The substrate is protected by a photolithographic mask using the resist AZ40XT'T . Afterwards a seed layer stack consisting of $50 \mathrm{~nm}$ chrome and $200 \mathrm{~nm} \mathrm{NiFe}$ is applied. Following a photolithographic mask is applied defining the ring core's structure. The $6 \mu \mathrm{m}$ ring core consisting of $\mathrm{NiFe} 45 / 55$ is electroplated. After removing of the resist, the $\mathrm{NiFe}$ seed layer is removed as well by ion beam etching. The $\mathrm{NiFe} 45 / 55$ core is shown in Figure 3.

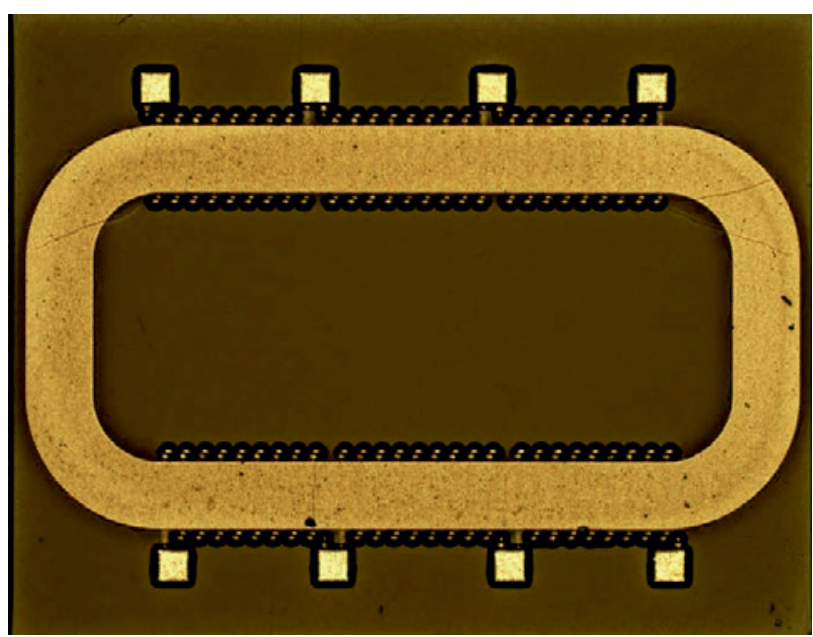

Figure 3. Deposited NiFe45/55 core

Following a $50 \mathrm{~nm}$ chromium and $200 \mathrm{~nm}$ gold seed layer is deposited in order to apply the Vias by cooper electroplating. As the structure has been filled with copper and the seed layer has been removed, the Vias and the ring core are embedded in Durimide. Upon the exposed Vias the upper layer of coils is structured. The coils are closed by electroplated copper shown in Figure 4.

Simultaneously the contact pads are raised. In a third embedding step the completed micro transformer (shown in Figure 5) is insulated against external environmental influences.

Finally, the completed wafer was separated into chips by dicing. 


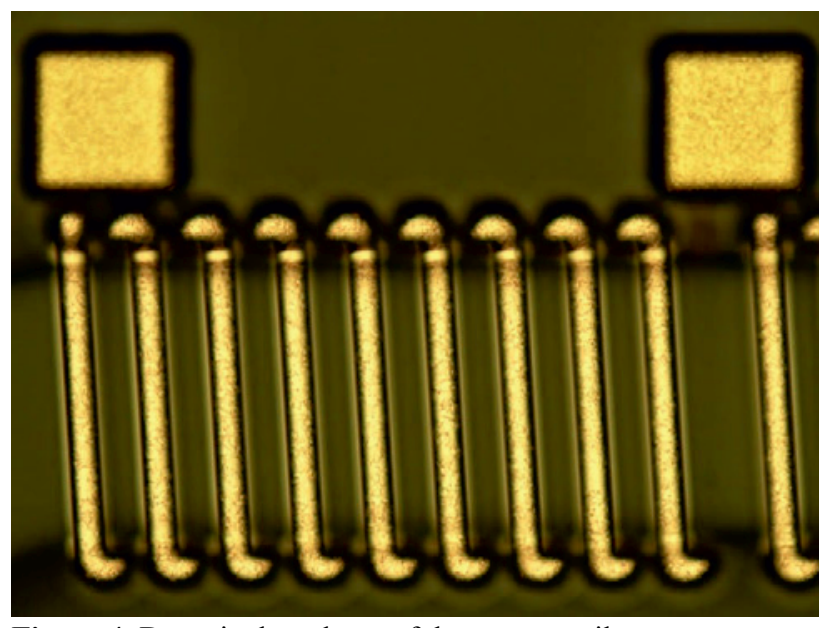

Figure 4. Deposited top layer of the copper coils.

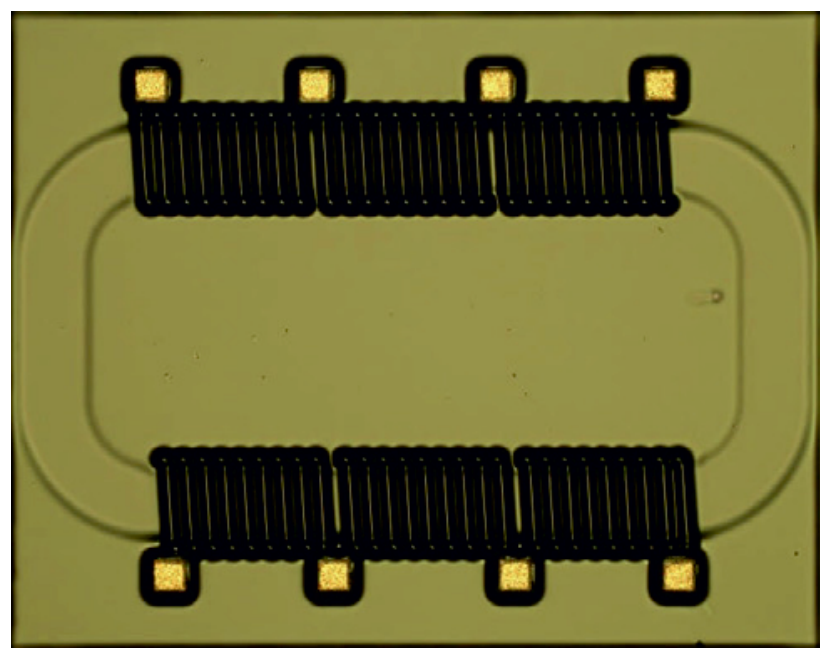

Figure 5. Complete insulated micro transformer.

\section{Results}

Except of an ohmic resistance (Rdc) measurements, we don't have at the moment a possibility to perform other electrical measurements on device level. Therefore, the some separated micro transformer devices were mounted in an open cavity QFN package. The bonding procedure is performed manually with an Ultra-Sonic-Bond process. In the Figure 6 is shown a prepared microtransformer device for testing.

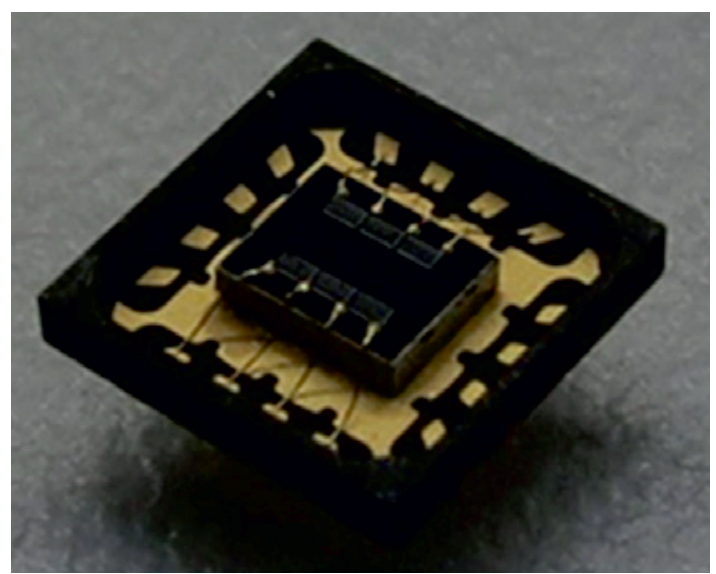

Figure 6. Microtransformer packaged into QFN housing
As a QFN housing was chosen a smallest, adequate, and on the market available housing. The QFN housing $5 \mathrm{~mm} \times 5 \mathrm{~mm}$ with 16 leads was applied. For our tests only 8 leads were used.

The measured Rdc of one coil is about $250 \mathrm{~m} \Omega$. Other measurements like an inductivity and Q-factor were measured applying Agilent Impedance Analyzer E4991A. The devices were measured with a signal of $5 \mathrm{~mA}$ at $1 \mathrm{MHz}$. A single coil shows an inductance of about $12 \mathrm{nH}$. Two serial connected coils have an inductance of $24 \mathrm{nH}$, were three serial connected coils have an inductance of $37 \mathrm{nH}$ (Figure 7). The inductance is stable up to $30 \mathrm{MHz}$.

For exact measurement of an inductance a measurement method is under development. With actual method the parasitic inductance of a couple of $\mathrm{nH}$ was also measured and we don't received the real inductance of the microtransformer.

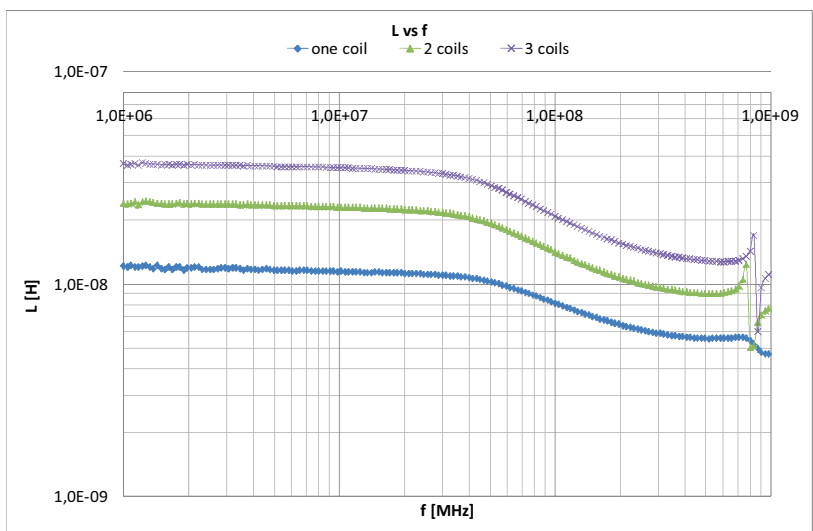

Figure 7. Measurement Inductance vs. Frequency

Maximal Q factor is measured at frequency of $20 \mathrm{MHz}$ and has a value of 3 . All coils (single coil, two and three serial connected coils) show the same Q factor.

Figure 8 shows the characteristic of resistance of a transformer coils as a function of frequency.

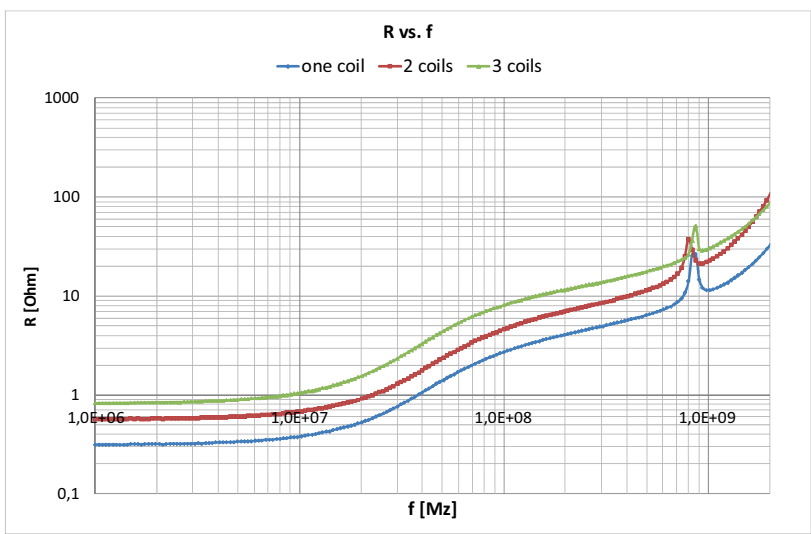

Figure 8. Measurement Resistance vs. Frequency

Also the DC-Bias characteristic of transformer coils was measured. This result is shown in the Figure 9. DC-Bias measurement was measured using Wayne Kerr 3260B. For this measurement was also same oscillating level of $5 \mathrm{~mA}$ at $1 \mathrm{MHz}$ applied. Maximal DC-Bias of $500 \mathrm{~mA}$ was applied. At DC-Bias of $250 \mathrm{~mA}$ decrease the initial 
inductance of microtransformer coil to the value of $50 \%$ of the initial inductance $(\Delta L / L=50 \%)$.

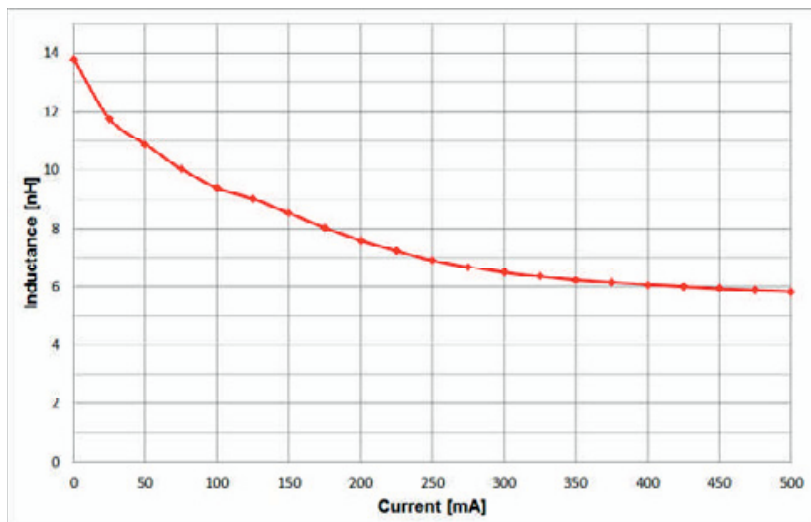

Figure 9. DC-Bias characteristic of one transformer coil

\section{References}

1. R. Foley et al, Applied Power Electronics Conference and Exposition (APEC) 2010, 525 (2010)
2. A.W. Lofti et al., $7^{\text {th }}$ International Conference on Integrated Power Electronics Systems (CIPS) 2012, Nuremberg, Germany, (2012)

3. T. Liakopoulos, A. Panda, M. Wilkowski, A. Lofti, 3rd International Workshop on Power Supply on Chip (PwrSoC 2012), San Francisco, USA, (2012)

4. Patent application WO2013072135 (A1)

5. M.C. Wurz, D. Dinulovic, H.H. Gatzen, Proc. 8th Int. Symposium on Magnetic Materials, Processes and Devices, 206th Meet. of The Electrochemical Society 2004, Honolulu, Hawaii, USA, (2004)

6. C. Ruffert, J. Chen, L. Rissing, European Congress and Exhibition on Advanced Materials and Processes (Euromat 2011), Montpellier, France, (2011)

7. C. Ruffert, H. H. Gatzen, Microsystem Technologies, 14, 9-11, (2008)

8. M. G. Allen, IEEE Trans. On Magnetics, 39, 5-2, (2003) 